\title{
Widely Tunable Twin-Guide Laser Diodes With Sampled Gratings: Design and Performance
}

\author{
René Todt, Thomas Jacke, Ralf Meyer, Reinhard Laroy, Geert Morthier, Senior Member, IEEE, \\ and Markus-Christian Amann, Senior Member, IEEE
}

\begin{abstract}
The widely tunable twin-guide laser diode with sampled gratings is a monolithically integrated device that utilizes a vertical integration scheme to realize an electronically and widely tunable distributed feedback (DFB)-type laser. Owing to its DFBtype structure, it possesses many advantages, such as large continuous tuning ranges, simple wavelength tuning scheme, and promising dynamic characteristics. In this paper, we detail on the design of the sampled grating tunable twin-guide laser and discuss its performance. Recent devices achieve tuning ranges over $40 \mathrm{~nm}$ along with high side-mode suppression $(\geq 35 \mathrm{~dB})$ as well as large output power $(\geq 10 \mathrm{~mW})$.
\end{abstract}

Index Terms-Distributed feedback (DFB) lasers, laser tuning, optical communication, semiconductor lasers, wavelength division multiplexing.

\section{INTRODUCTION}

$\mathbf{T}$ HE INCREASING demand for transmission capacity over optical fiber cables necessitates an efficient use of the already deployed fibers. Widely tunable lasers with tuning ranges of at least $40 \mathrm{~nm}$ are key components to this end, as they enable dynamic wavelength provisioning and routing. Over the past years, a considerable number of device concepts for widely tunable lasers has been presented (for an overview see Ref. [1] and [2]), such as monolithically integrated tunable laser diodes based on sophisticated gratings [3]-[5], lasers based on ring resonators [6], distributed feedback (DFB) laser arrays [7], microelectromechanical systems (MEMS) vertical-cavity surfaceemitting lasers (VCSELs) [8], and miniaturized external cavity lasers [9]. While there is presently no single technology that outperforms all others, monolithically integrated widely tunable lasers are certainly among the promising concepts as they offer high output power, high spectral selectivity, and fast switching times on the order of several nanoseconds.

Manuscript received October 31, 2006; revised July 9, 2007. This work was supported in part by the European Union via the Information Society Technologies (IST) Project IST-2000-28244 "NEWTON" and in part by the Institute for the Promotion of Innovation by Science and Technology in Flanders (IWT) via a specialisation grant for R. Laroy.

R. Todt was with Walter Schottky Institut, Technische Universität München, Garching D-85748, Germany. He is now with the Nano Electronics Research Laboratories, NEC Corporation, Ibaraki 305-8501, Japan (e-mail: r-todt@ bx.jp.nec.com).

T. Jacke was with Walter Schottky Institut, Technische Universität München, D-85748 Garching, Germany.

R. Meyer, and M.-C. Amann are with Walter Schottky Institut, Technische Universität München, D-85748 Garching, Germany (e-mail: mcamann@ wsi.tum.de).

R. Laroy and G. Morthier are with the Department of Information Technology, Ghent University, Ghent B-9000, Belgium (e-mail: geert.morthier@ intec.UGent.be).

Color versions of one or more of the figures in this paper are available online at http://ieeexplore.ieee.org.

Digital Object Identifier 10.1109/JSTQE.2007.904172
A drawback of the monolithically integrated widely tunable lasers, which have traditionally been distributed Bragg reflector (DBR)-type lasers, has been their rather complex tuning schemes, requiring three or even more tuning currents to set the emission wavelength. Progress on this issue has only recently been achieved by the invention of several widely tunable DFB-like lasers that do not require a phase tuning section anymore [10]-[12], and hence, require only a total of two tuning currents, which is expected to dramatically facilitate the device characterization and control. At present, the only DFB-type widely tunable laser that combines electronic tunability and competitive state-of-the-art performance is the sampled grating (SG) tunable twin-guide (TTG) laser diode [11], [13].

In this paper, we will detail on the design of widely tunable SG-TTG laser diodes, discuss their performance, and outline strategies for even further improvements of their performance characteristics.

\section{DeVice STRUCTURE}

The SG-TTG laser, as depicted in Fig. 1, is a further development of the original DFB-TTG laser diode [14]. In essence, the TTG laser consists of two $p-i-n$ heterojunction diodes forming active and tuning region. These two low-bandgap regions are electronically decoupled by a high-bandgap $n$-InP separation layer, thereby allowing to bias both sections independently. All regions possess a common $n$-contact that is placed laterally beneath the buried ridge, and the current flow into the active and tuning region is controlled via the potential applied to the frontand backside- $p$-contacts, respectively.

From an optical point of view, the buried ridge forms a singlemode waveguide that confines significant portions of the optical field in the active as well as in the tuning layer, as described by large confinement factors $\Gamma_{\mathrm{a}}$ and $\Gamma_{\mathrm{t}}$ [Fig. 1(c)]. In contrast to DBR-type tunable lasers, the TTG laser is a vertically integrated device. One of the benefits of the vertical integration scheme is that the roundtrip phase is invariant against longitudinally homogeneous refractive index changes brought about by injecting carriers into the tuning regions to tune the wavelength-selective grating. Thus, a separate phase tuning section is not necessary in the TTG laser.

To achieve wide wavelength tuning, the tuning region is split in the longitudinal direction into two sections. Both of them contain SGs that possess comb-like reflection spectra of slightly different periodicity, which allows for extending the tuning range by utilizing the Vernier effect [15]. Differential tuning of the reflectors (i.e., tuning one reflector while leaving the other one unchanged) results in large wavelength jumps, referred to as 


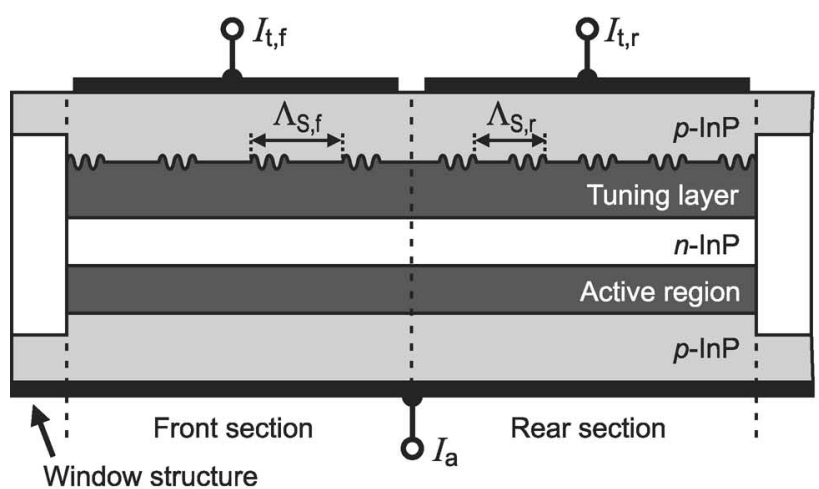

(a)

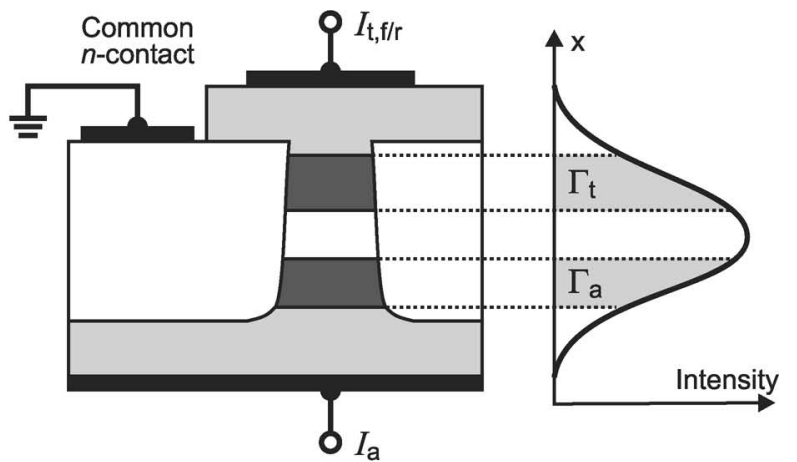

(b) (c)

Fig. 1. Schematic structure of a widely tunable SG-TTG laser diode. (a) Longitudinal cross-section. (b) Lateral cross-section. (c) Mode distribution.

supermode hops, which are typically on the order of $5-7 \mathrm{~nm}$ depending on the specific grating design. On the other hand, simultaneous tuning of both reflectors results in a continuous wavelength shift just like in a DFB-TTG laser.

\section{GRATING Design}

As for any monolithic widely tunable laser, the gratings are among the key elements of the SG-TTG laser design. Even for the rather simple SGs, the design space is considerable and includes:

1) the reflection peak spacing $P_{\mathrm{f} / \mathrm{r}}$ in the comb-like front and rear reflector (which is determined from the sampling period $\Lambda_{\mathrm{S}, \mathrm{f} / \mathrm{r}}$ according to $\left.P_{\mathrm{f} / \mathrm{r}}=\lambda^{2} / 2 n_{\mathrm{g}} \Lambda_{\mathrm{S}, \mathrm{f} / \mathrm{r}}\right)$ as well as the peak spacing difference $\Delta P=\left|P_{\mathrm{f}}-P_{\mathrm{r}}\right|$,

2) the sampling duty cycle $\delta_{\mathrm{f} / \mathrm{r}}$, which is the fraction of the sampling period containing a grating,

3 ) the coupling coefficient $\kappa_{0}$ of the grating, and

4) the length of the front and rear reflector $L_{\mathrm{f} / \mathrm{r}}$.

While the influence of the various design parameters on the optical properties of the device can be qualitatively anticipated in many cases, a thorough understanding is greatly facilitated by exploring the design space quantitatively. In the following, we will therefore, investigate the influence of the aforementioned design parameters on the tuning range and side-mode rejection (quantified in terms of the normalized end-loss difference $\left.\Delta \alpha_{\mathrm{m}} L\right)$ of the SG-TTG laser.

The first part of this section will focus on basic SG configurations, which are characterized by approximately equally long front and rear reflectors $\left(L_{\mathrm{f}} \cong L_{\mathrm{r}}\right)$ and equal sampling duty cycles $\left(\delta_{\mathrm{f}} \cong \delta_{\mathrm{r}}\right)$. Accordingly, we will refer to these gratings as symmetric reflector designs. Subsequently, the benefits of more advanced asymmetric reflector designs will be briefly discussed in the second part of this section.

The simulation results shown in the following section were obtained from a transfer-matrix-based threshold analysis method. It should be noted that the impact of spurious facet reflections, which was discussed in a previous paper [16], has been neglected in the present analysis by assuming ideal antireflection (AR) coatings.

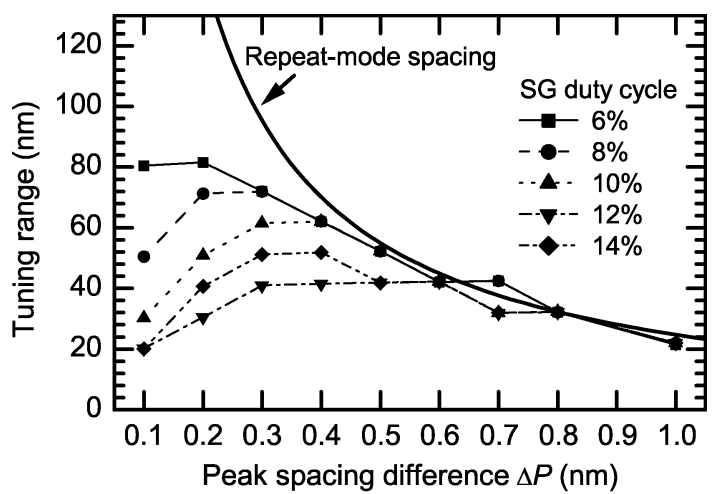

(a)

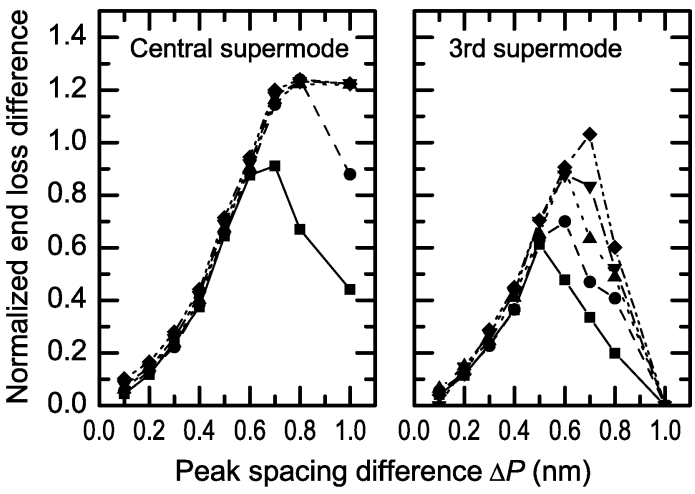

(b)

Fig. 2. Tuning range (a) and normalized end loss difference $\Delta \alpha_{\mathrm{m}} L$ (b) vs. peak spacing difference $\Delta P$ with sampling duty cycle $\delta$ as parameter. Simulation parameters: $P_{\mathrm{f}}=5.0 \mathrm{~nm}, P_{\mathrm{r}}=5.1-6.0 \mathrm{~nm}, \Delta P=0.1-$ $1.0 \mathrm{~nm}, \delta=6-14 \%$, effective $\kappa L$-value $\kappa_{\text {eff, }, 0} L=1.0$, device length $L=$ $1200 \mu \mathrm{m}, \lambda / 4$-phase shift between front and rear reflector.

\section{A. Symmetric Reflector Designs}

1) Peak Spacing Difference and Sampling Duty Cycle: The peak spacing difference $\Delta P$ and the sampling duty cycle $\delta$ are the two most important design parameters. Fig. 2 illustrates how they influence the achievable tuning range and the normalized end loss difference $\Delta \alpha_{\mathrm{m}} L$.

At large peak spacing differences $(\Delta P \geq 0.6 \mathrm{~nm})$, the tuning range is limited by the repeat mode spacing, independently of 
the sampling duty cycle. With decreasing peak spacing difference, the tuning range is at first increasing, then saturates, and subsequently, decreases again. The specific value of $\Delta P$, where the saturation and the following decrease occurs, depends on the sampling duty cycle $\delta$. This is a consequence of the nonflat envelope of the SG reflection spectrum, which decreases as $\sin (x) / x$ when moving away from the central reflection peak. Neglecting the phase condition, lasing takes place at the wavelength where the aggregate grating reflectivity is largest. For a combination of two slightly different comb-like reflectors with perfectly flat envelope this is always at the position where reflection peaks of both reflectors are lined up. However, in an SG, competition takes place between the aligned reflection peak pair and slightly misaligned reflection peak pairs that are situated closer to the center wavelength of the grating. As the latter ones possess a higher reflectivity, it may well happen that their aggregate reflectivity is larger than that of the aligned reflection peak pair, and thus, the tuning range becomes limited by the bandwidth of the SG. This competition becomes stronger with decreasing peak spacing difference $\Delta P$ as this enhances the overlap, and therefore, also the aggregate reflectivity of the misaligned reflection peaks. Furthermore, as the bandwidth of an SG decreases with increasing sampling duty cycle, devices with large sampling duty cycle are considerably more affected by this effect.

From the simulations, a sampling duty cycle of up to $14 \%$ appears acceptable to obtain the desired $40 \mathrm{~nm}$ tuning range, at least under the assumption of a flat gain curve. However, in practice, a nonflat gain curve will additionally decrease the tuning range, and therefore, some additional margin should be included in the device design, imposing an upper limit of $\sim 10-12 \%$ for the sampling duty cycle.

The tuning range given in Fig. 2(a) is not sufficient to fully assess the quality of a grating design because it does not reveal any information on the provided spectral selectivity. Therefore, Fig. 2(b) depicts the dependence of the normalized end loss difference $\Delta \alpha_{\mathrm{m}} L$ on the peak spacing difference $\Delta P$ for the central supermode and the third-order supermode. Due to the envelope of the SG, the central supermode provides the largest end loss difference, which decreases slightly with increasing supermode order. As the third-order supermodes are typically the highest order supermodes that need to be accessed to cover a 40 nm tuning range, the two plots in Fig. 2(b) represent the upper and lower limits of $\Delta \alpha_{\mathrm{m}} L$ that would be encountered within the aforementioned tuning range. As a rule of thumb, a normalized end loss difference of 0.1 is sufficient to obtain a side-mode suppression ratio (SMSR) of $30 \mathrm{~dB}$ at an output power of $1 \mathrm{~mW}$ for a typical conventional, i.e. not widely tunable, laser diode [17]. However, as will be illustrated in Section IV, depending on the flatness of the active region gain characteristic, a considerably larger end loss difference may be required in a widely tunable device.

The normalized end loss difference of the central supermode increases at first with the peak spacing difference independently of the sampling duty cycle. Differences become apparent only at larger values of $\Delta P(\geq 0.7 \mathrm{~nm})$ : for large sampling duty cycle $(\delta=10-14 \%)$, the end loss difference monotonically increases up to a saturation value, indicating that the peak spacing differ- ence $\Delta P$ has become larger than the width of the individual reflection peaks. For small sampling duty cycle $(\delta=6-8 \%)$, a decrease is observed that is caused by the repeat supermode. With decreasing repeat mode spacing (respectively increasing $\Delta P$ ), the repeat supermode moves more and more into spectral regions where significant reflection is provided by the $\mathrm{SG}$, gains intensity, and finally, limits the spectral selectivity. As the bandwidth of the SG is approximately inversely proportional to the sampling duty cycle, devices that utilize SGs with small duty cycle are more susceptible to this effect, which then becomes dominant already at smaller peak spacing differences.

The behavior observed for the third-order supermode is qualitatively the same as for the central supermode. More specifically, the normalized end loss difference increases until the spectral selectivity becomes limited by the repeat supermode and subsequently decreases. As the third-order supermode is situated in considerable spectral distance from the center wavelength of the $\mathrm{SG}$, it is more prone to the influence of the repeat supermode. Therefore, at any given sampling duty cycle, the rollover takes place already at lower values of $\Delta P$ and is also observed for large sampling duty cycles. As long as the spectral selectivity is limited by a neighboring supermode, the normalized end loss difference of the third-order supermode is only insignificantly smaller than for the central supermode.

In light of the aforementioned considerations, a sampling duty cycle of $10 \%$ along with a peak spacing difference between 0.4 and $0.6 \mathrm{~nm}$ seems to be particularly well suited for an aspired tuning range of $40 \mathrm{~nm}$. The corresponding normalized end loss difference of $\Delta \alpha_{\mathrm{m}} L=0.4-0.9$ appears to be more than sufficient to ensure stable single-mode operation even in consideration of a nonflat gain curve. Furthermore, the sampling duty cycle is, on the one hand, small enough to enable a tuning range of $40-60 \mathrm{~nm}$, while, on the other hand, it is still large enough to prevent the repeat supermode from impairing the spectral selectivity.

2) $\kappa L$-Value: The $\kappa L$-product is a key design parameter of any DFB-type laser diode. Apart from its significance for yield issues and spatial hole burning-related effects, which are not considered at this point, the $\kappa L$-value influences the spectral selectivity.

In the SG-TTG laser, the normalized end loss difference exhibits a broad maximum at $\kappa L$-values of around 1.0. Insufficient optical feedback reduces the spectral selectivity at lower $\kappa L$ values, whereas the broadening of the individual SG reflection peaks leads to an increased overlap of the misaligned reflection peak pairs and causes a reduction of the end loss difference at $\kappa L$-values above 2.0.

The optimum $\kappa L$-value is considered to be in the range between 1.0 and 1.5. Not only is the end loss difference highest in that region, but additionally, as in the case of fixed-wavelength DFB laser diodes [18], spatial hole burning is also expected to be minimal there.

3) Device Length: Typically, the device length of conventional DFB laser diodes is rather short, on the order of $300-600 \mu \mathrm{m}$. Thus, the cavity mode spacing is large, and consequently, enables excellent side-mode suppression. The situation is, however, slightly different in a Vernier-effect-based tunable 


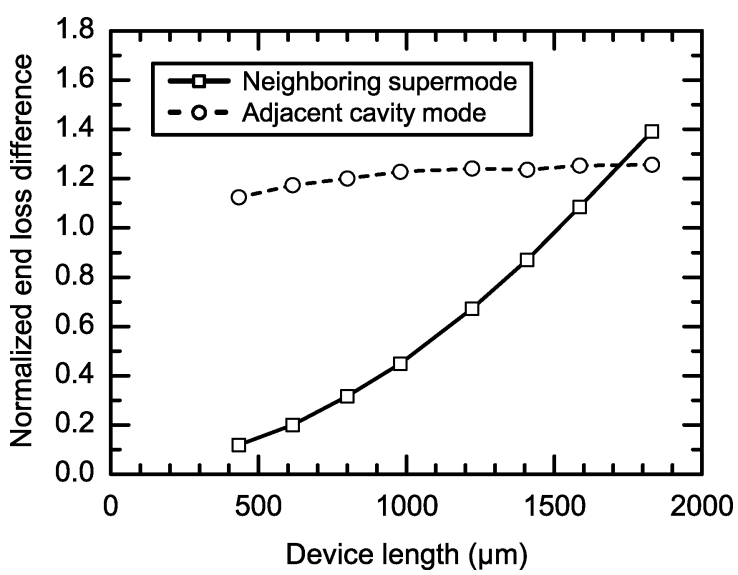

Fig. 3. Normalized end loss difference with respect to the neighboring supermode and the adjacent cavity mode as function of the device length. Simulation parameters: $P_{\mathrm{f}}=5.0 \mathrm{~nm}, P_{\mathrm{r}}=5.5 \mathrm{~nm}, \Delta P=0.5 \mathrm{~nm}, \delta=10 \%$, effective $\kappa L$-value $\kappa_{\text {eff, } 0} L=1.0$, device length $L=430-1830 \mu \mathrm{m}, \lambda / 4$ phase shift between front and rear reflector.

laser. Usually, its spectral purity is limited by a neighboring supermode and not by an adjacent cavity mode, and therefore, the fabrication of longer devices is beneficial as will be discussed later.

Fig. 3 depicts the normalized end loss difference with respect to the neighboring supermode and the adjacent cavity mode in function of the device length. In practice, the mode with the lowest end loss difference will limit the side-mode rejection. In the present example, this is the neighboring supermode as long as the device length remains below $\sim 1700 \mu \mathrm{m}$, and only above this length, the adjacent cavity mode becomes the dominant side mode.

The normalized end loss difference $\Delta \alpha_{\mathrm{m}} L$ (with respect to the neighboring supermode) increases, approximately linearly with the device length. This is because the SG reflection peaks become more narrow, and in turn, the overlap of the reflection peaks of the neighboring supermode decreases, which ultimately enhances the corresponding end loss difference.

The optimum side-mode rejection is obtained when the end loss difference of the neighboring supermode and the adjacent cavity mode are equal, which would be at a device length of $\sim 1700 \mu \mathrm{m}$ for the specific example considered here. This illustrates the general need for comparatively long devices.

4) Phase Shift: In the absence of facet reflections, any DFB-type laser without phase shift possesses two dominant cavity modes with equal end loss. Consequently, such a device shows multimode operation. The use of a $\lambda / 4$ phase shift is one solution to this issue that is commonly employed for fixedwavelength DFB laser diodes and brings about single-mode operation with ideally two equally strong side modes.

Fig. 4 reveals that the incorporation of a $\lambda / 4$ phase shift in between the two SG reflectors of a widely tunable SG-TTG laser has just the same effect on the mode spectrum as in the case of a fixed-wavelength DFB laser. While two dominant cavity modes (at 1549.8 and $1550.2 \mathrm{~nm}$ ) with equal end loss $\left(26.5 \mathrm{~cm}^{-1}\right)$ are found in the non-phase-shifted device, only one is found for the $\lambda / 4$ phase-shifted laser (at $1550.0 \mathrm{~nm}$ with an end loss

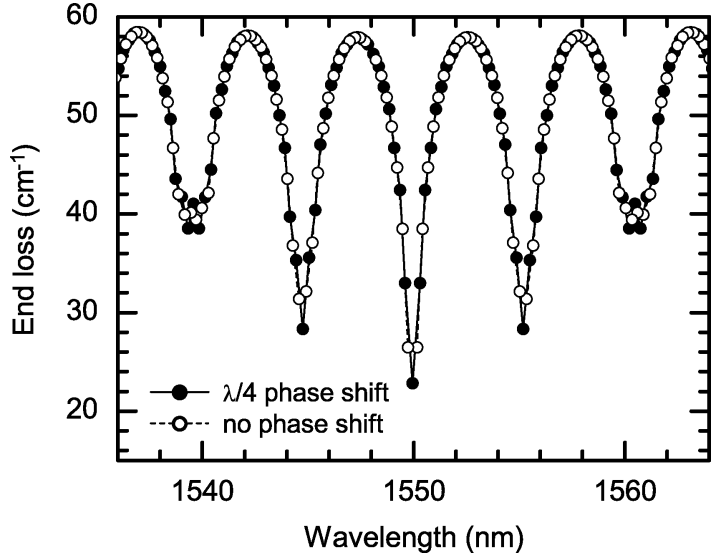

Fig. 4. Mode spectrum of a $\lambda / 4$ phase-shifted (solid symbols) and a non-phase-shifted (open symbols) SG-TTG laser. Cavity modes are represented by dots while lines are shown only as a visual aid. Simulation parameters: $P_{\mathrm{f}}=5.0 \mathrm{~nm}, P_{\mathrm{r}}=5.5 \mathrm{~nm}, \Delta P=0.5 \mathrm{~nm}, \delta=10 \%$, effective $\kappa L$-value $\kappa_{\text {eff }, 0} L=1.0$, device length $L=1200 \mu \mathrm{m}$.

of $22.8 \mathrm{~cm}^{-1}$ ). It should be noted that the suppression of the neighboring cavity mode is almost independent of the phase shift length, which mainly influences the suppression of the adjacent cavity mode. While increasing the phase shift length from 0 to $\lambda / 4$, the latter one increases approximately linearly. In this respect, the SG-TTG behaves similar to a fixed-wavelength DFB laser, and thus, the precise control of the phase shift length appears to be important.

\section{B. Asymmetric Reflector Designs}

A drawback of the SG-TTG laser is its need for AR-coated facets on both sides of the device. Employing a symmetric reflector design, about half of the output power is lost at the rear facet, which limits the maximum power that can be coupled into a fiber. Therefore, concepts to increase the output power fraction at the front facet are of interest. Asymmetric reflector designs are very useful to this end. Even though we have not yet employed such designs in practice, we will briefly outline their potential in the following by discussing one particularly simple but nevertheless very effective example.

In the present example, the front reflector sampling duty cycle was lowered from an initial value of $10 \%$ to a minimum value of $3 \%$ while the rear reflector sampling duty cycle was increased simultaneously by the same amount and all other device parameters were left unaltered. Thus, the reflectivity of the rear mirror was enhanced whereas that of the front mirror was lowered, thereby raising the output power at the front facet. Fig. 5 reveals the extent by which the output power fraction at the front facet is enhanced and how the spectral selectivity is affected. The fraction of the output power at the front facet increases from initially $48 \%$ (the initial output power fraction at the front facet is not equal to $50 \%$ because usually even the so-called symmetric reflector designs possess a very slight asymmetry) to up to $90 \%$. However, at the same time, the end loss difference is decreasing, at first only slowly and insignificantly but rapidly and pronounced in the end. 


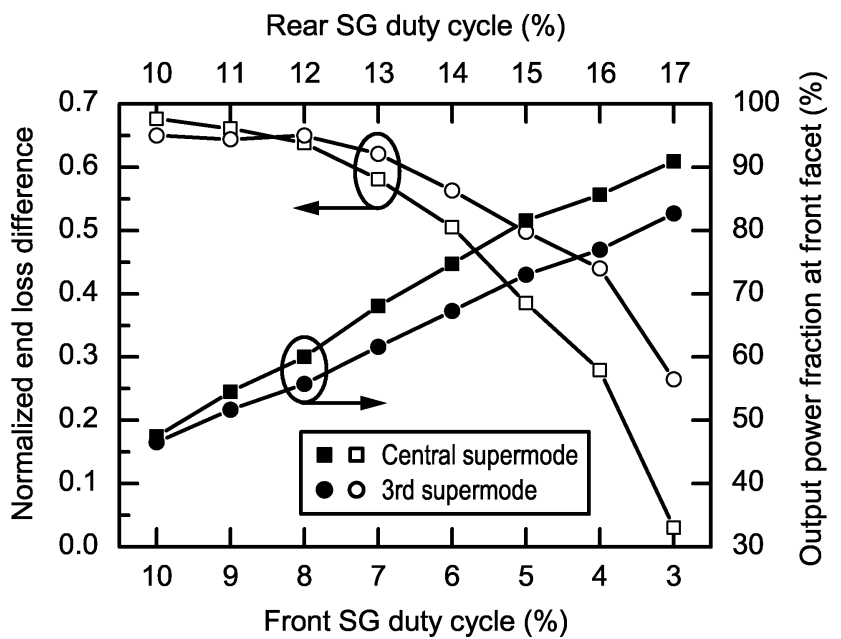

Fig. 5. Effect of unequal front and rear reflector sampling duty cycles: normalized end loss difference and fraction of output power at front facet vs. sampling duty cycle. Simulation parameters: $P_{\mathrm{f}}=5.0 \mathrm{~nm}, P_{\mathrm{r}}=5.5 \mathrm{~nm}, \Delta P=$ $0.5 \mathrm{~nm}$, front reflector sampling duty cycle $\delta_{\mathrm{f}}=3-10 \%$, rear reflector sampling duty cycle $\delta_{\mathrm{r}}=10-17 \%$, effective $\kappa L$-value $\kappa_{\text {eff, } 0} L=1.0$, device length $L=1200 \mu \mathrm{m}(600 \mu \mathrm{m}$ per reflector), $\lambda / 4$ phase shift between front and rear reflector.

The stronger increase of the output power fraction of the central supermode as compared to the third-order supermode is related to the nonflat envelope of the SG reflection peaks. Two counteracting effects have to be taken into account. Increasing the sampling duty cycle of the rear reflector enhances its reflectivity. However, at the same time, its bandwidth also decreases. While this decrease in bandwidth is irrelevant for the central supermode, it results in a less strong increase of the third-order reflection peak. For similar arguments, the normalized end loss difference of the third-order supermode decreases less pronounced than that of the central supermode.

A reduction of the normalized end loss difference from 0.65 to 0.5 , as observed by reducing the front reflector sampling duty cycle from $10 \%$ to $6 \%$, appears to be acceptable. In fact, for a sufficiently flat gain characteristic of the active region, probably even a further reduction of the normalized end loss difference could be feasible without significantly impairing the spectral purity. In any case, a reduction of the front reflector sampling duty cycle to $6 \%$ (along with the corresponding increase of the rear reflector sampling duty cycle) raises the fraction of the output power at the front facet to $\sim 70 \%$, which constitutes a relative increase by more than $40 \%$. Considering the simplicity of this approach, such a performance enhancement is remarkable and highlights the large potential that lies within asymmetric mirror designs.

\section{DEVICE PERformance}

Successful realization of an SG-TTG laser fully covering a tuning range of more than $40 \mathrm{~nm}$ was first reported in [13], along with the details on its fabrication process. In this section, we will summarize the performance characteristics of the SG-TTG lasers and evaluate their performance in light of the design considerations given in the preceding section.
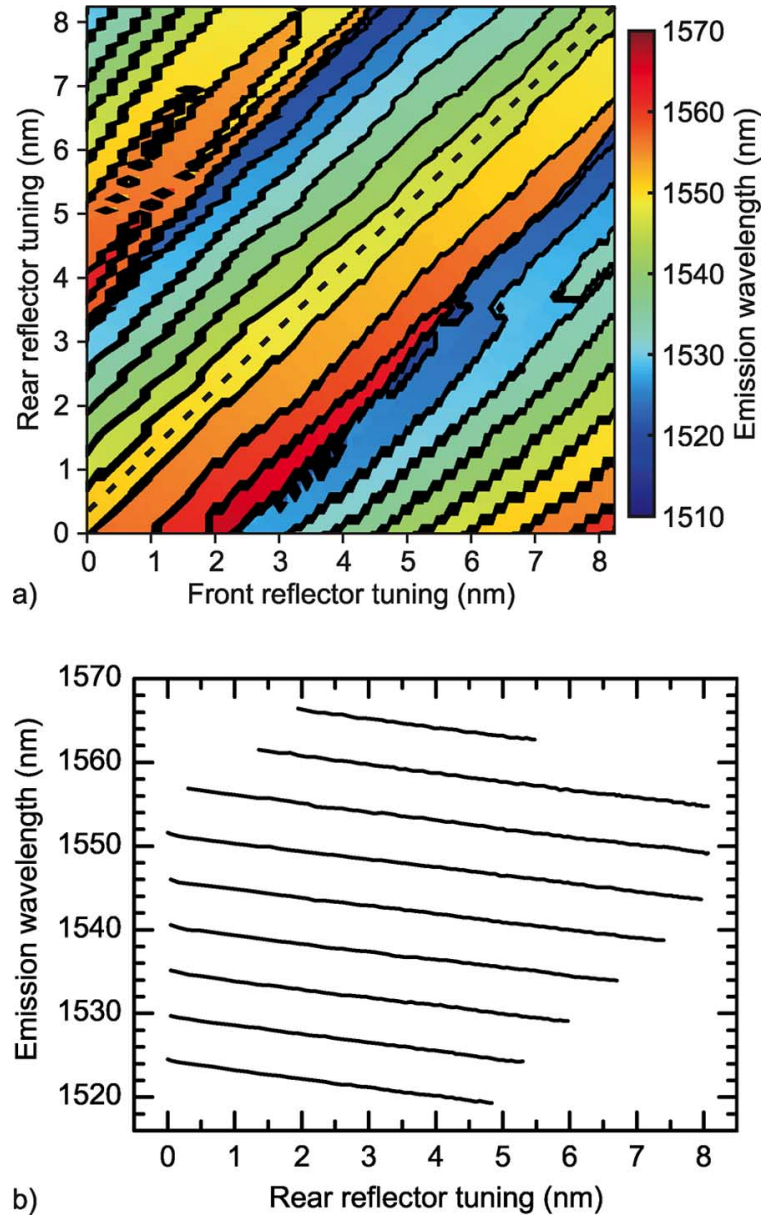

Fig. 6. (a) Wavelength map of an SG-TTG laser. The various supermodes are enclosed by solid black lines. Within each supermode, the wavelength changes continuously and mode-hop-free by up to $8.2 \mathrm{~nm}$. The dashed line indicates an exemplary tuning curve discussed in detail in Fig. 7. (b) Emission wavelength vs. rear reflector tuning for the main supermodes as extracted from (a). Note that both reflectors have to be tuned simultaneously to obtain the depicted tuning curves.

The devices discussed here are $1200 \mu \mathrm{m}$ in length and feature a symmetric mirror design with a peak spacing of $P_{\mathrm{f}}=$ $5.0 \mathrm{~nm}$ and $P_{\mathrm{r}}=5.5 \mathrm{~nm}$ and a sampling duty cycle of $\delta=$ $10 \%$. The effective $\kappa L$-value of the central supermode was estimated to be $\sim 1.1$. Devices with and without phase shift were evaluated, and contrary to theoretical predictions, no significant differences were found between them. All of the data presented in the following was obtained from a non-phase-shifted laser. The characterization was carried out at a submount temperature of $20^{\circ} \mathrm{C}$ and at an active region current of $I_{\mathrm{a}}=250 \mathrm{~mA}$.

Fig. 6 illustrates the wavelength tuning characteristics of a typical SG-TTG laser. The wavelength map shown in Fig. 6(a) was obtained by sweeping both tuning currents in a nonlinear way up to a maximum current of $45 \mathrm{~mA}$. Subsequently, the tuning currents were converted to the wavelength shift of the reflector to allow for a better visualization of the data. The wavelength map reveals the location of the various supermodes and clearly proves the absolutely regular tuning behavior of the SG-TTG laser. The central supermode (indicated as dashed line) is surrounded by a total of eight main supermodes: three situated on the long-wave side and five on the short-wave side of 


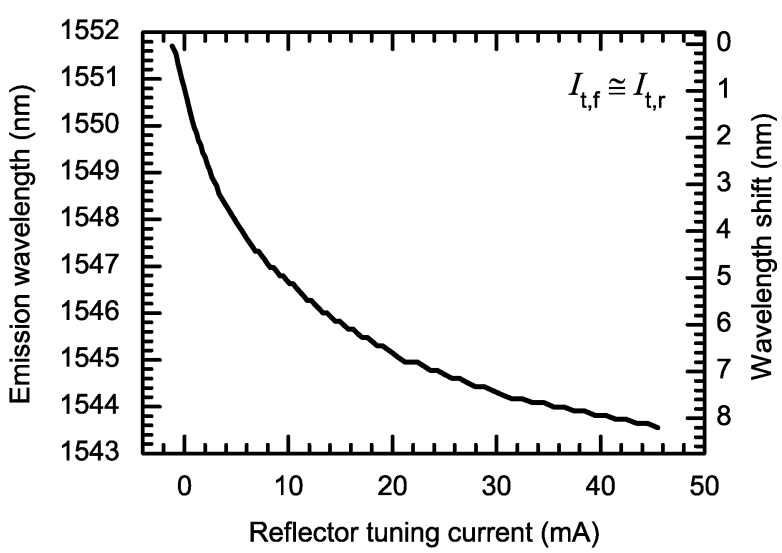

(a)

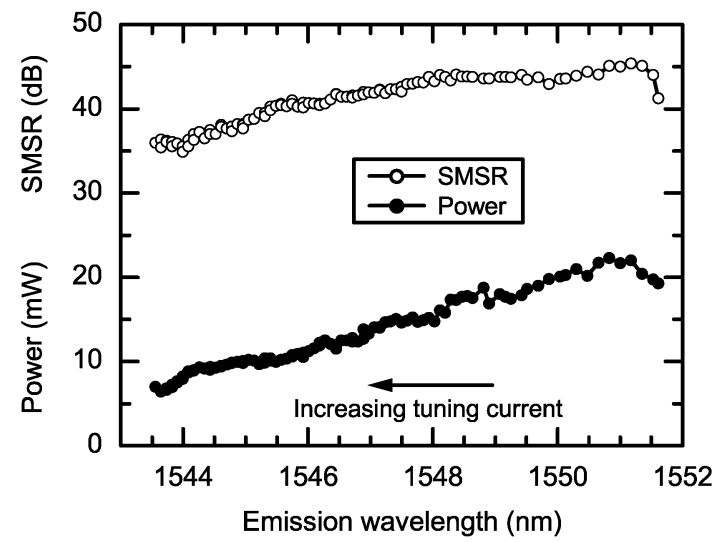

(b)

Fig. 7. Tuning characteristics of the central supermode (indicated as dashed line in Fig. 6(a), demonstrating continuous tuning over an 8.2 nm-large wavelength range. (a) Emission wavelength and wavelength shift vs. tuning current. The tuning currents for the front and rear reflector are approximately equal. (b) Variation of ex-facet output power and SMSR as function of the emission wavelength. The tuning currents increase from right to left.

the central supermode. The asymmetry between the number of supermodes on the long- and short-wave side is a consequence of the nonflat gain characteristic as will be pointed out later.

For the main supermodes, the emission wavelength as function of the rear reflector tuning is shown in Fig. 6(b). The spacing of the supermodes as well as the wavelength change within each of the supermodes is absolutely regular. The continuous tuning range of the supermodes varies between 5 and $8 \mathrm{~nm}$. Only the supermode located around $1565 \mathrm{~nm}$ has a considerably smaller tuning range of $3.7 \mathrm{~nm}$. In contrast to all other supermodes, its tuning range is not limited by saturation of the wavelength shift in the front or rear reflector, but by strong changes in the gain characteristics that lead to a supermode jump from the longwave to the short-wave side of the tuning range. For all other supermodes, the continuous tuning range is given by the continuous tuning range of the central supermode $(\sim 8 \mathrm{~nm})$ minus the amount of differential tuning required to switch to the corresponding supermode. Hence, moving further away from the central supermode, the continuous tuning range becomes smaller.

Additional details on the tuning characteristics are presented in Fig.7, which exemplarily depicts the wavelength change as well as the variations of output power and SMSR while tuning

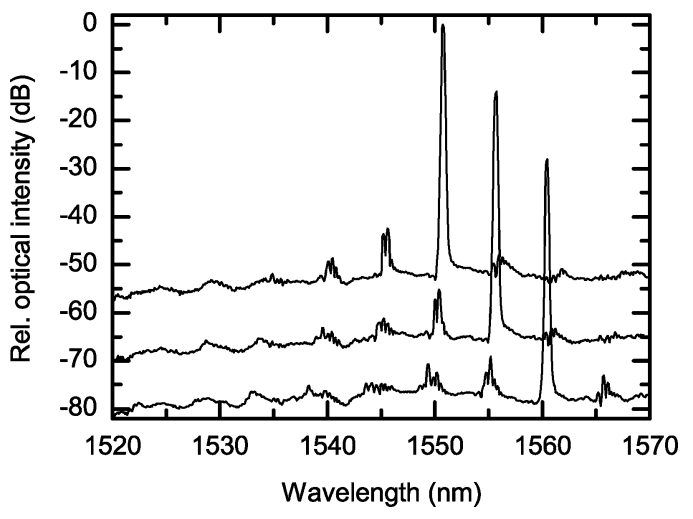

Fig. 8. Optical emission spectra of three neighboring supermodes, demonstrating the high spectral purity of the SG-TTG laser. The SMSR is limited by neighboring supermodes.

along the central supermode. As can be seen, the emission wavelength shifts continuously from 1551.7 to $1543.5 \mathrm{~nm}$, thereby covering an $8.2 \mathrm{~nm}$ tuning range. The output power decreases almost linearly from 22 to $7 \mathrm{~mW}$ with increasing wavelength shift. Similarly, the SMSR decreases smoothly from a maximum value of 45 to $35 \mathrm{~dB}$ at the end of the tuning range. While the SMSR is expected to scale with the output power, the pronounced decrease is not typical for TTG lasers and can only be explained to a limited extent by the output power variation. A substantial portion of the decrease was attributed to changes in the gain profile during tuning.

Typical optical emission spectra illustrating the high spectral purity of the SG-TTG laser are shown in Fig. 8. The periodicity of the SG reflection peaks and the corresponding supermodes are clearly recognizable in the spectra. As usual for lasers employing Vernier effect tuning, the SMSR is limited by reflections due to the nonvanishing overlap of neighboring SG reflection peaks. Within the resolution limit of the optical spectrum analyzer, no adjacent cavity mode is discernible. This is, to some extent, surprising because the specific device presented here does not contain a phase shift, and thus, would be expected to show multimodal behavior with two approximately equally strong adjacent cavity modes. Owing to the very low facet reflectivity of about $10^{-4}$, it appears unlikely that reflections from the facets can remove the mode degeneracy. Therefore, it is presumably the rather complicated SG structure along with inherent fabrication variations that brings about enough asymmetry to lift the degeneracy of the modes in the AR-coated laser. As mentioned previously, no significant differences were found between devices with and without phase shift. Even though no detailed statistical analysis was conducted, the large number of devices investigated allows for a rough estimate on the single-mode yield. Out of about 30 investigated lasers, only 1 device showed multimodal behavior. This finding suggests that the single-mode yield is at least $90-95 \%$. Compared to AR-coated DFB laser diodes [18], such a high single-mode yield is certainly surprising and appears as a remarkable feature of the SG-TTG laser.

The optical emission spectra shown in Fig. 8 also reflect once more the influence of the nonflat gain characteristic. The SMSR is, in many cases, limited by neighboring SG reflection 


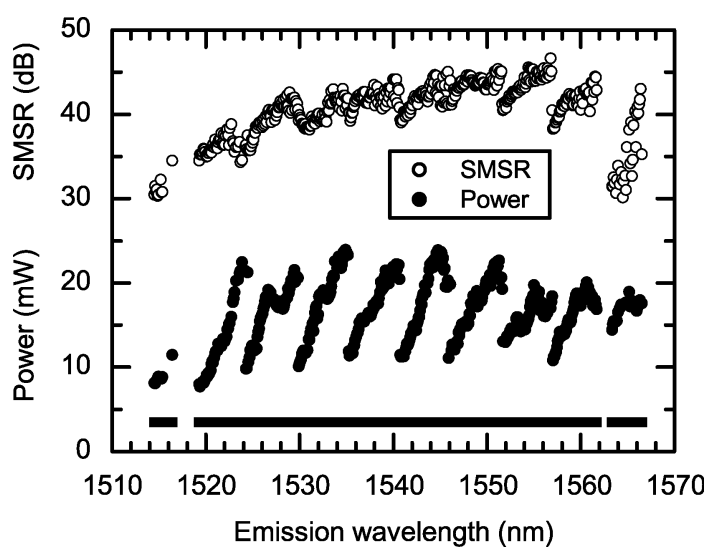

Fig. 9. Ex-facet output power and SMSR vs. emission wavelength for the main supermodes. The bold horizontal line at the bottom of the graph is shown as a visual guide to easily locate gaps within the tuning range. A wavelength range of more than $40 \mathrm{~nm}$ is fully covered with SMSR $\geq 35 \mathrm{~dB}$ and output power $\geq 10 \mathrm{~mW}$.

peaks on the short-wave side of the main mode. Even though the tuning currents can be adjusted to obtain equally strong side modes on either side, the SMSR was observed to be lower in this case. A more quantitative assessment of the influence of the gain curve was possible by measuring the gain characteristic using the Hakki-Paoli method. The measurements revealed a pronounced increase of the slope $(d g / d \lambda)$ of the gain characteristic in combination with a shift toward the short-wave limit of the tuning range during tuning. By taking the slope into account for computing the normalized end loss difference, we find that $\Delta \alpha_{\mathrm{m}} L$ decreases from $\sim 0.6$ (assuming a flat gain curve) to $\sim 0.1$. This explains well for the untypically strong variations of the SMSR and the slight asymmetry observed in the alignment of the main supermodes with respect to the central one. Hence, with respect to the SMSR, we expect that substantial improvements are possible by utilizing an optimized active region design, or by increasing the device length from the presently used $1200 \mu \mathrm{m}$ (dictated by technological limitations) to the optimum length of $1700 \mu \mathrm{m}$.

The static performance characteristics of the SG-TTG laser are summarized in Fig. 9 showing SMSR and output power versus emission wavelength. As can be seen, the SMSR and output power remain above $35 \mathrm{~dB}$ and $10 \mathrm{~mW}$, respectively, over a wavelength range of $41 \mathrm{~nm}$ (from 1520.5 to $1561.5 \mathrm{~nm}$ ).

Also, with respect to the output power level, small modifications seem to hold a quite significant potential for even further improvements. Firstly, we mention here an improved lateral current injection scheme that was demonstrated for DFB-TTG lasers [19]. It is technologically easy to implement, is suitable for the SG-TTG laser, and is expected to effectively reduce shunt currents. Secondly, we refer to the benefits of the asymmetric reflector designs discussed in the previous section. From rough estimates, these modifications should allow for an output power increase by about a factor of 2 .

Finally, it should also be noted that the SG-TTG laser has promising prospects for direct modulation [20]. From parasitic free relative intensity noise measurements, a maximum intrinsic bandwidth in excess of $20 \mathrm{GHz}$ as well as a bandwidth of up to $12 \mathrm{GHz}$ at typical operation conditions of $I_{\mathrm{a}}=250 \mathrm{~mA}$ was deduced. These values represent some of the highest that have ever been reported for widely tunable laser diodes and are a benefit of the all-active device structure of the TTG laser. It remains, however, to be shown whether parasitics can be sufficiently reduced and whether the thermal properties of the TTG structure indeed allow for a direct modulation at such high frequencies.

\section{CONCLUSION}

We have discussed the design and performance of widely tunable TTG laser diodes with SGs. The SG-TTG lasers, which fully cover a wavelength range of more than $40 \mathrm{~nm}$ with high spectral purity (SMSR $\geq 35 \mathrm{~dB}$ ) and large output power $(\geq 10 \mathrm{~mW})$, possess performance characteristics comparable to the more established longitudinally integrated multisection DBR lasers. The large continuous tuning ranges of the SG-TTG laser as well as its straightforward wavelength control requiring only two tuning currents are unmatched hitherto and greatly simplify the device characterization. Additionally, the device design of the SG-TTG laser was shown to still hold significant potential for even further improvements of the performance characteristics. Hence, the SG-TTG laser is one of the most promising monolithically integrated widely tunable laser diodes.

\section{ACKNOWLEDGMENT}

The authors greatly appreciate the technical assistance of J. Grottenthaler, L. Mora, R. Heilmann, and E. Sckopke.

\section{REFERENCES}

[1] J. Buus, M.-C. Amann, and D. J. Blumenthal, Tunable Laser Diodes and Related Optical Sources, 2nd ed. Piscataway, NJ: Wiley-IEEE Press, 2005.

[2] L. A. Coldren, G. A. Fish, Y. Akulova, J. S. Barton, and C. W. Coldren, "Tunable semiconductor lasers: A tutorial," J. Lightw. Technol., vol. 22, no. 1, pp. 1-202, Jan. 2004.

[3] G. A. Fish, "Multi-electrode tunable laser," in Proc. SPIE, 2001, vol. 4532 pp. 37-40.

[4] J.-O. Wesström, S. Hammerfeldt, J. Buus, R. Siljan, R. Laroy, and H. D. Vries, "Design of a widely tunable modulated grating Y-branch laser using the additive Vernier effect for improved super-mode selection,' in Proc. IEEE Int. Semicond. Laser Conf., Garmisch-Partenkirchen, Germany, 2002, pp. 99-100.

[5] D. C. J. Reid, D. J. Robbins, A. J. Ward, N. D. Whitbread, P. J. Williams, G. Busico, A. C. Carter, A. K. Wood, N. Carr, J. C. Asplin, M. Kearley, W. Hunt, D. R. Brambley, and J. Rawsthorne, "A novel broadband DBR laser for DWDM networks with simplified quasi-digital wavelength selection," in Proc. Opt. Fiber Commun. Conf., Los Angeles, CA, 2002, pp. 541-543.

[6] M. Takahashi, Y. Deki, S. Takaesu, M. Horie, M. Ishizaka, K. Sato, K. Kudo, K. Suzuki, T. Kaneko, X. Xu, and H. Yamazaki, "A stable widely tunable laser using a silica-waveguide triple-ring resonator," in Proc. Opt. Fiber Commun. Conf., Anaheim, CA, 2005, Paper PDP19.

[7] H. Hatakeyama, K. Naniwae, K. Kudo, N. Suzuki, S. Sudo, S. Ae, Y. Muroya, K. Yashiki, K. Satoh, T. Morimoto, K. Mori, and T. Sasaki, "Wavelength-selectable microarray light sources for S-, C-, and L-band WDM systems," IEEE Photon. Technol. Lett., vol. 15, no. 7, pp. 903-905, Jul. 2003.

[8] M. Maute, B. Kögel, G. Böhm, P. Meissner, and M.-C. Amann, "MEMS tunable 1.55- $\mu \mathrm{m}$ VCSEL with extended tuning range incorporating a buried tunnel junction," IEEE Photon. Technol. Lett., vol. 18, no. 5, pp. 688-690, Feb. 2006

[9] K. Sato, J. De Merlier, K. Mizutani, S. Sudo, S. Watanabe, K. Tsuruoka, K. Naniwae, and K. Kudo, "Over 50-mW fiber coupled power and 40-nm 
tuning range of an extremely compact external cavity wavelength tunable laser," in Proc. Eur. Conf. Opt. Commun., Glasgow, UK, 2005, Paper Th2.5.2

[10] H. Ishii, Y. Kondo, F. Kano, and Y. Yoshikuni, "A tunable distributed amplification DFB laser diode (TDA-DFB-LD)," IEEE Photon. Technol. Lett., vol. 10, no. 1, pp. 30-32, Jan. 1998.

[11] G. Morthier, B. Moeyersoon, and R. Baets, "A $\lambda / 4$-shifted sampled or superstructure grating widely tunable twin-guide laser," IEEE Photon. Technol. Lett., vol. 13, no. 10, pp. 1052-1054, Oct. 2001.

[12] R. Todt, T. Jacke, R. Meyer, and M.-C. Amann, "Thermally widely tunable laser diodes with distributed feedback," Appl. Phys. Lett., vol. 87, no. 2, p. 021103, Jul. 2005.

[13] R. Todt, T. Jacke, R. Meyer, J. Adler, R. Laroy, G. Morthier, and M.-C. Amann, "Sampled grating tunable twin-guide laser diodes with over 40-nm electronic tuning range," IEEE Photon. Technol.Lett., vol. 17, no. 12, pp. 2514-2516, Dec. 2005.

[14] M.-C. Amann, S. Illek, C. Schanen, and W. Thulke, "Tunable twin-guide laser: A novel laser diode with improved tuning performance," Appl. Phys. Lett., vol. 54, no. 25, pp. 2532-2533, Jun. 1989.

[15] V. Jayaraman, Z.-M. Chuang, and L. A. Coldren, "Theory, design, and performance of extended tuning range semiconductor lasers with sampled gratings," IEEE J. Quantum Electron., vol. 29, no. 6, pp. 1824-1834, Jun. 1993.

[16] R. Todt and M.-C. Amann, "Influence of facet reflections on monolithic widely tunable laser diodes," IEEE Photon. Technol.Lett., vol. 17, no. 12, pp. 2520-2522, Dec. 2005.

[17] G. Morthier and P. Vankwikelberge, Handbook of Distributed Feedback Laser Diodes. Norwood, MA: Artech House, 1997.

[18] K. David, G. Morthier, P. Vankwikelberge, R. G. Baets, T. Wolf, and B. Borchert, "Gain-coupled DFB lasers versus index-coupled and phaseshifted DFB lasers: A comparison based on spatial hole burning corrected yield," IEEE J. Quantum Electron., vol. 27, no. 6, pp. 1714-1723, Jun. 1989.

[19] R. Todt, T. Jacke, R. Meyer, and M.-C. Amann, "High output power tunable twin-guide laser diodes with improved lateral current injection structure," Electron. Lett., vol. 41, no. 4, pp. 190-191, Feb. 2005.

[20] R. Laroy, R. Todt, R. Meyer, M.-C. Amann, G. Morthier, and R. Baets, "Direct modulation of widely tunable twin-guide lasers," IEEE Photon. Technol. Lett., vol. 18, no. 12, pp. 1293-1295, Jun. 2006.

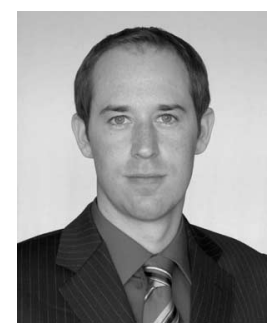

René Todt was as born in Miltenberg, Germany, in 1977. He received the M.Sc. degree in physics from the State University of New York, Albany, USA, in 2001 and the Dr. rer. nat. degree in physics from the Technical University of Munich, Munich, Germany, in 2006.

From 2001 to 2005 he was with Walter Schottky Institute, Technical University of Munich, where he was engaged in the research of monolithically integrated wavelength tunable laser diodes. In 2006, he joined the System Platforms Research Laboratories, NEC Corporation, Tokyo, Japan, where he is involved in the research and development of wavelength tunable lasers and related optoelectronic devices. $\mathrm{He}$ is the author or coauthor of over 40 articles published in scientific journals and conference proceedings.

Dr. Todt is a member of the German Physical Society (DPG).

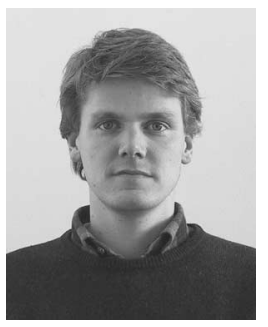

Thomas Jacke received the Diploma in physics from the Technical University (RWTH) Aachen, Aachen, Germany, in 1999, and the Ph.D. degree from the Technical University of Munich, Munich, Germany, in 2007.

In 1999-2000, he was engaged in the research of positron-based medical imaging techniques in the Research Center (FZ) Jülich. From 2001 to 2005, he was with Walter Schottky Institute, Technical University of Munich, where he was involved in the research of monolithically integrated tunable laser diodes. In 2006 , he joined a major automotive supplier, where he is engaged in the development of silicon-based bipolar power semiconductors. He authored or coauthored over 30 articles on tunable laser diodes in scientific journals and conference proceedings.

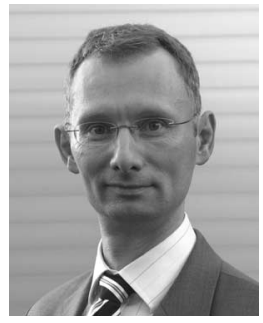

Ralf Meyer was born in Oldenburg, Germany, in 1963. He received the Diploma in physics from Rheinisch-westfälische Technische Hochschule (RWTH) Aachen, Aachen, Germany, in 1990, and the $\mathrm{Ph} . \mathrm{D}$. degree in physics from the Research Center Jülich, Jülich, Germany, in 1994.

He was with the Department of Electrical Engineering, University of Kassel, Kassel, Germany, where he collaborated in the establishing of a research group and in a leading position in the founding of the Institute for Microstructure Technology and Analytics. In 1998, he became the Head of the III-V Technology Group, Walter Schottky Institute, Technical University of Munich, Munich, Germany, where he is engaged in the development and realization of a variety of different types of light emitters in the wavelength range from 1 to $10 \mu \mathrm{m}$ based on III-V compound semiconductors. He participated in several national and European projects. He is the author or coauthor of about 50 publications. 2003.

Dr. Meyer organized the German Workshop on Molecular Beam Epitaxy in

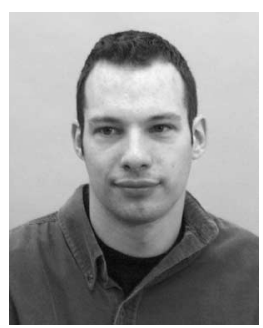

Reinhard Laroy received the degree in electrical engineering and the Ph.D. degree in electrical engineering from Ghent University, Ghent, Belgium, in 2001 and 2006, respectively.

From August 2001 to February 2006, he was with the Department of Information Technology (INTEC), Ghent University. He is currently working at the Belgian Institute for Postal services and Telecommunications, Brussels, Belgium, as a Technical Advisor in Telecom Regulations.

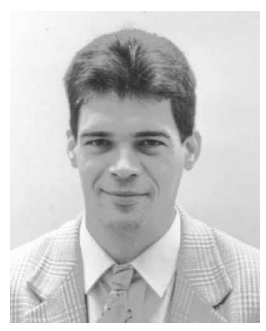

Geert Morthier (SM'00) received the degree in electrical engineering and the Ph.D. degree in electrical engineering from the University of Ghent, Ghent, Belgium, in 1987 and 1991, respectively.

From September 1987 until August, he served the Belgian army. Since 1991, he has been a member of the permanent staff of IMEC. His current research interests include the modeling and characterization of optoelectronic components. He is the author or coauthor of over 100 published papers in the field and holds several patents. He is also the coauthor of the Handbook of Distributed Feedback Laser (Artech House, 1997). From 1998 to 1999, he was the Project Manager of the ACTS project ACTUAL dealing with the control of widely tunable laser diodes, and from 2001 to 2005, he was the Project Manager of the IST project NEWTON on new widely tunable lasers. In 2001, he was appointed a parttime Professor at Ghent University, where he teaches courses on optical fiber communication and lasers. He is the Editor of the IEE Proceedings Optoelectronics.

Dr. Morthier has served on the program committee of several international conferences. 


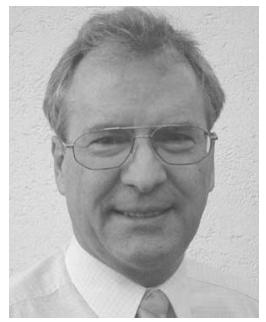

Markus-Christian Amann (SM'00) was born in Singen/Hohentwiel, Germany, in 1950. He received the Diplom and Dr.-Ing. degrees from the Technical University of Munich, Munich, Germany, in 1976 and 1981, respectively, both in electrical engineering.

From 1981 to 1994, he was with the Corporate Research Laboratories, Siemens AG, Munich, where he was involved in the research on long-wavelength InGaAsP-InP laser diodes, and in 1990, he became the Deputy Director for the research on laser diodes and integrated optoelectronic devices. In 1994, he joined the Department of Electrical Engineering, University of Kassel, Kassel, Germany, as a Full Professor for technical electronics establishing a working group for III/V semiconductor electronics and optoelectronics. Since November 1997, he has been the Chair of the Semiconductor Technology at Walter Schottky Institute, Technical University of Munich, where he is currently engaged in the research on tunable laser diodes for the near-infrared, quantum cascade lasers, long-wavelength vertical-cavity laser diodes, and laser diode applications. He is the author or coauthor of over 200 articles (including some 30 invited) on semiconductor optoelectronics published in scientific journals conference proceedings, and two books.

Dr. Amann is a member of the German Informationstechnische Gesellschaft (ITG) and a Senior Member of the IEEE Lasers and Electro-Optics Society. He has served on numerous conference committees such as the IEEE Semiconductor Laser Conferences, the Indium Phosphide and Related Materials Conferences, and the Conferences on Lasers and Electro-Optics. 